\title{
Geotechnical data aggregation and visualisation supporting informed risk management: the one-stop geotech shop
}

\author{
SDN Wessels Rio Tinto Iron Ore, Australia
}

R Dixon Rio Tinto Iron Ore, Australia

\begin{abstract}
Rio Tinto Iron Ore (RTIO) operates 16 mine operations in the Pilbara region of Western Australia. At each operation, there could be many active pits that pose a challenge for the effective management of geotechnical risks with limited resources. There are many information sources which the geotechnical engineer uses for risk management, including temporal and non-temporal data that may or may not be georeferenced. This information can be in various places, such as proprietary software, network servers or local devices. Consolidating this information can be time-consuming for the engineer and may not include all available data sources. To address this, RTIO developed an in-house tool for aggregating and visualising data, where all geotechnical data can be accessed in a single platform and also included a dashboard to provide visibility of monitoring status and risk profiles across all RTIO Pilbara operations. Sustainability of an in-house developed tool poses business challenges and a decision was made to explore available external options. Although a number of off-the-shelf applications were identified, none met all the RTIO requirements. The best-fit application was ultimately selected with RTIO working with the vendor to incorporate all defined software requirements. This paper discusses RTIO expectations and requirements for the data aggregation and visualisation application, challenges relating to the in-house system development, the evaluation process for a vendor managed option and development of the application functionality with the selected vendor.
\end{abstract}

Keywords: geotechnical risk management, slope monitoring, data aggregation, visualisation, multi-pit operations

\section{$1 \quad$ Background}

Approximately 1,200 km north of Perth, Rio Tinto Iron Ore (RTIO) operates 16 iron ore operations in the Pilbara region of Western Australia (Figure 1). The RTIO mines and associated port and rail infrastructure delivered 338 Mt of iron ore to the market in 2018. The 'Pilbara Challenge' related to the development and implementation of systems due to the large number of operations and pits (over 100 active pits at any time), geographical spread, significant planned expansions and limited geotechnical resources has been described by de Graaf \& Wessels (2016) and Wessels \& Dixon (2019). RTIO geotechnical resources include Perth-based engineers accountable for geotechnical investigations and designs for greenfield and brownfield projects, and site-based engineers accountable for design implementation, monitoring and operational risk management.

The focus of this paper is the consolidation of the systems used by the site geotechnical engineer for monitoring and operational risk management. At any one operation, the site geotechnical team may comprise 2-4 geotechnical engineers working a fly-in-fly-out (FIFO) roster of eight days on and six days off. The team may be managing slope hazards for up to 10 operational pits, in addition to stockpiles and waste dumps. The number of slopes and individual monitoring data sources pose a significant challenge for effective management of geotechnical risks. Information sources used by the geotechnical engineer for risk management include temporal and non-temporal data that may or may not be georeferenced. For example, the slope monitoring for a site may include two radar units, seven automated and nine manual prism pillars with over 1,200 prisms, five-time domain reflectometry (TDR) cables, and 60 vibrating wire piezometer monitoring devices. Other data sources include logging and mapping data, geotechnical reports and documents, photographs and slope risk assessments. Information can be stored in various places, such as 
proprietary software, network servers or local devices. Consolidating this information can be time-consuming for the engineer and there is the risk that information may be excluded and not all available data sources will be reviewed together.

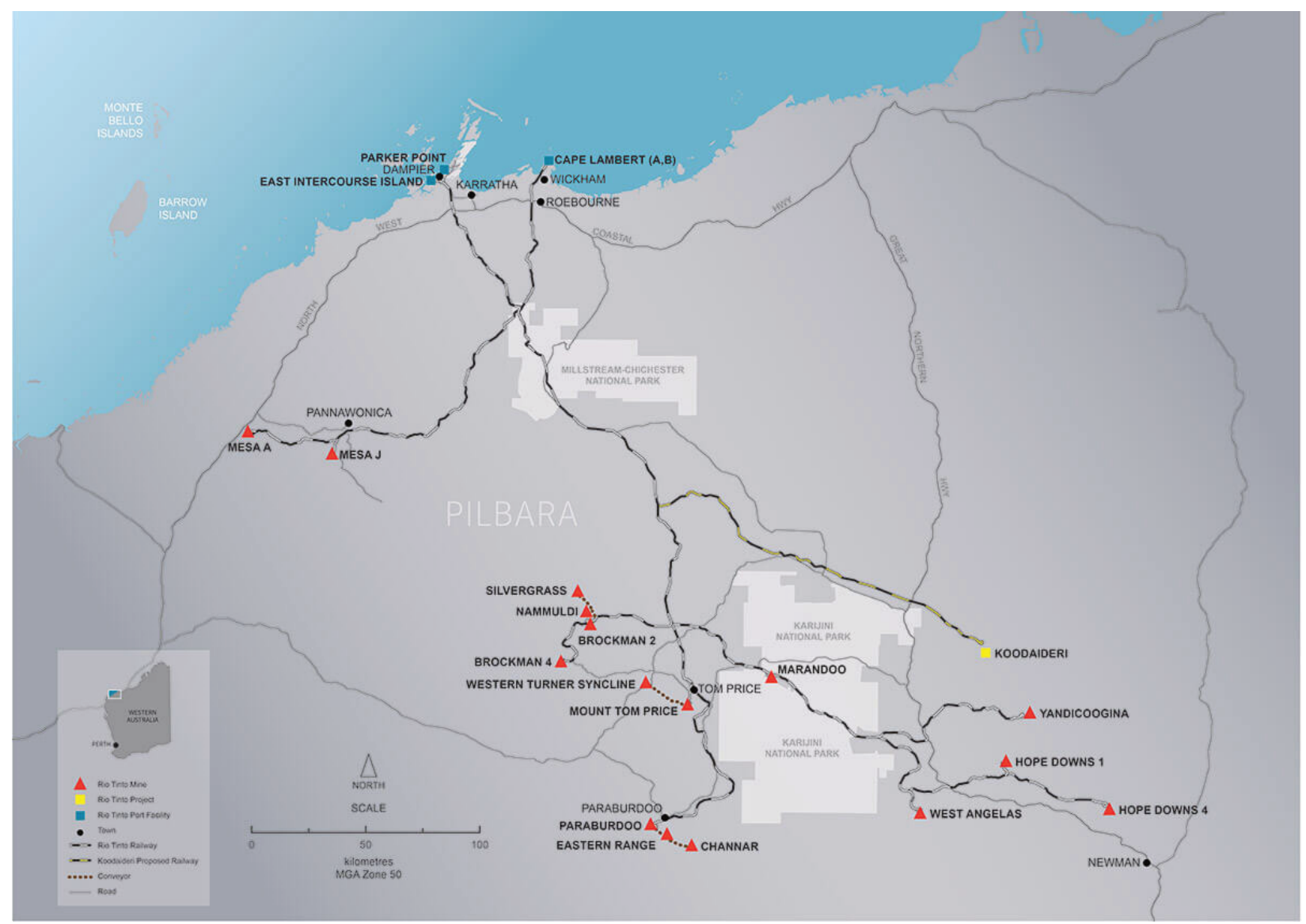

Figure 1 Location map of the Pilbara operations, Western Australia

\section{Geotechnical risk management information}

Effective geotechnical risk management includes consideration of data and observations from both slope monitoring and slope performance systems to validate and update design inputs. The importance of diligent slope monitoring to detect signs of failure and protect exposed mine personnel has been described in several case studies (Girard \& McHugh 2000; Girard 2001; Sjöberg 1996). Sjöberg (1996) outlined the importance of slope monitoring programs to maintain safe operations and provide information on slope behaviour and warning of instability. In addition, the components of a comprehensive monitoring plan have been described and may include "instruments capable of measuring rock mass displacement, groundwater parameters, and blast vibration" (Girard 2001).

Whilst slope monitoring data is temporal in nature, slope performance focusses more on non-temporal information. Examples of which include survey of physical conformance to design, observations of fall of ground events and geotechnical hazards, and general pit slope inspections. Although these slope performance parameters have a temporal component, for the purpose of this paper, we define temporal data as data with an ongoing time series, and non-temporal data as data that is collected on an ad hoc or routine basis but is not reported as time series plots. Ongoing observation and documentation of slope performance, in the form of maps and reports, is also an important input into the design feedback loop for remedial and optimisation slope designs. 


\subsection{Georeferenced temporal data sources}

RTIO use a risk-based approach to slope monitoring, ensuring appropriate monitoring controls are implemented commensurate with risk (de Graaf \& Wessels 2016). Slopes that are considered low risk may require infrequent berm inspections and manual monitoring techniques. Monitoring frequency is increased with risk level and near-real-time telemetry and alarming is used for high and critical risk slopes. Central to the risk-based approach is the Geotechnical Risk and Hazard Management System (GRAHAMS), to assess and capture geotechnical risks for pits and dumps. The current risk (risk with any existing controls in place) is evaluated using the GRAHAMS tool and where the risk level is unacceptable, additional layers of monitoring controls are introduced (Wessels \& Dixon 2019). Multiple monitoring devices and layers of monitoring employed for any one slope results in many data sources used by the geotechnical engineer for risk management.

Georeferenced temporal data sources used within RTIO include both surface and subsurface monitoring installations. Surface displacement monitoring includes automated and manual prism monitoring using Trimble 4D and radar systems from multiple vendors. Subsurface displacement monitoring includes TDR cables targeting the primary instability mechanism of structurally controlled sliding on shale and bedding, and inclinometers where rock mass controlled mechanisms are identified. Monitoring data for each instrument is available within the vendor proprietary software and databases (typically SQL or Microsoft Excel) stored on network servers or local devices. The geotechnical engineer uses the individual data sources for analysis and reporting via graphing of displacement and velocity. With engineers working on back-to-back 8-day roster panels, functionality that enables the saving of analysis views and reporting is critical to ensure thorough and consistent evaluation of data and communication to stakeholders. Other sources of georeferenced temporal data include pore pressure measurement using vibrating wire piezometers and weather information (i.e. rainfall) which is collated within EnviroSys.

Vendor software is also used for setting monitoring alarms depending on the level of monitoring. In RTIO, three levels of monitoring are defined: background (or baseline employed on all pit slopes, specifically low risk), diagnostic (applied to moderate risk with near-real time alarming), or critical (high risk areas where near-real-time alarmed monitoring must be in place for production to occur). Alarming status is transferred to the truck dispatch system located at the operations centre in Perth, where the pit controllers will respond to alarms as per trigger, action and response plans.

\subsection{Non-temporal information}

As part of a geotechnical engineer's workflow, non-temporal information related to a specific location in the pit (georeferenced) is often collected. Examples include slope performance documents such as visual inspections, fall of ground and hazard observations, wall mapping and geotechnical reconciliation reports.

Geotechnical hazards identified in the open pit are communicated to operations personnel through Geotechnical Hazard Alerts and Maps. The hazard alerts provide detail of the location of the realised hazards, the current risk to personnel working in the area and the additional controls required to mitigate the risk. For geotechnical engineers, it is also important to record and track the location of falls of ground, as these 'weak signals' (in the case of minor instabilities) can be a precursor of a large event that is developing.

RTIO use the in-house GRAHAMS tool to assess and capture geotechnical risks for pits and dumps. A key deliverable of GRAHAMS is defining the safety and economical risks to the business presented by geotechnical hazards. Several reports are available within GRAHAMS for communicating the risk profile to management and inclusion in mine plans. For spatial visualisation of geotechnical risk, a GRAHAMS risk summary is available within Google Earth Enterprise, where the highest safety and economic risks for each slope sector are presented as a series of coloured lights. 
Further types of georeferenced, non-temporal data include drilling, logging, testing and face mapping data available within acQuire and photographs which may be georeferenced if using a GPS camera. Non-georeferenced, non-temporal data sources used by the geotechnical engineer for risk management include documents as standards, procedures, guidance notes and templates.

\section{$3 \quad$ Monitoring data aggregation and visualisation}

Aggregating the many geotechnical data sources to facilitate interpretation and visualisation is a significant challenge faced by geotechnical engineers. The benefits of such a 'one-stop geotech shop' include removing the need for complex spreadsheets and standalone databases, removing human error, improvements in safety awareness, system growth capacity (new mines, expansions) and improved use of geotechnical engineer resources (Bar et al. 2018). An additional requirement for RTIO was to provide visibility of monitoring status and risk profiles across all RTIO Pilbara operations. Conrad \& Neuwirt (2019) outline how geotechnical monitoring platforms facilitate effective communication of risk and other key slope performance metrics to stakeholders assisting informed decision-making.

The RTIO need for developing a geoscience monitoring visualisation and management tool where all geotechnical data is aggregated and can be visualised in a single platform, was described by de Graaf \& Wessels (2013). At the time of development of the Geoscience Monitoring Data System (GMDS), no commercially available tool meeting all of RTIO's requirements existed. An in-house solution was hence developed, leveraging off prototype work completed at Rio Tinto's Kennecott Utah Copper Corporation. The primary benefit of the GMDS is providing a holistic picture of geotechnical slope performance giving the geotechnical engineer improved ability to understand, interpret and report on the diverse monitoring systems and non-temporal information. The GMDS is complementary to existing systems, not duplicating any (as shown in Figure 2).

The purpose of a data aggregator such as the GMDS is to provide a one-stop geotech shop to:

- Aggregate and display geotechnical information in a single platform that could work across the Pilbara operations.

- Collate, graph and display temporal monitoring data from various monitoring devices.

- Display a dashboard with summary health status for monitoring systems as a quick reference for geotechnical engineers and leaders.

- Show device locations in 2D and 3D.

- Integrate of geological, structural and 3D visualisation models, to assist with interpretation of monitoring data.

- Manage photographs and images, including the ability to access georeferenced images.

- Set and investigate monitoring alarms.

- Be able to create customised monitoring reports.

- Provide access to a variety of georeferenced and non-georeferenced geotechnical documents and reports, with longer-term strategy to use templates on a tablet in the field to update documents such as visual inspection reports and hazard alerts.

- Complement other RTIO geoscience databases (acQuire, EnviroSys, Vulcan) and RTVis and Google Earth Enterprise systems currently used for visualising geotechnical risk and hazard information. 


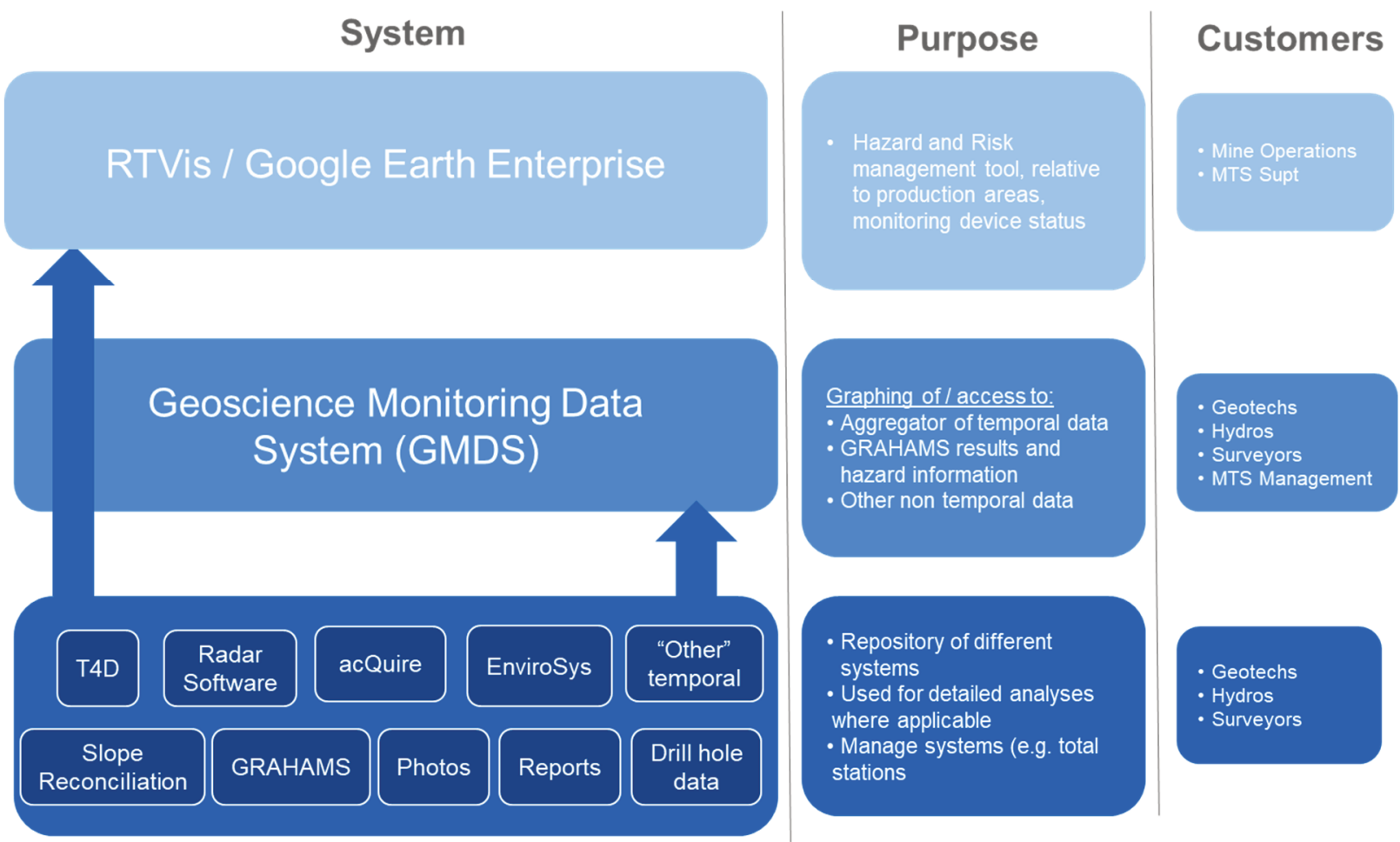

Figure 2 Rio Tinto Iron Ore geoscience monitoring systems, purpose, and customers

Between 2011 and 2015, development of the GMDS continued to approximately 80\% functionality, however, the system was cumbersome and relying on in-house resources for ongoing development and maintenance of the tool proved unsustainable. The concepts of an aggregator tool were proofed, but the product was to 'clunky' to deploy as a final product. One of the challenges that was experienced during the development of the GMDS was that the developers were not geotechnical engineers. When the RTIO team would submit a request for a feature to be developed, it would sometimes take a few iterations to come up with a product the reflected the original intent of the request. In 2015, vendor displays at the International Symposium on Slope Stability in Open Pit Mining and Civil Engineering in Cape Town showcased advances in this area and several off-the-shelf applications for geoscience monitoring visualisation. The products that were on display were much more developed, resulting in a more enjoyable and efficient user experience. This initiated a critical review of the RTIO in-house developed GMDS and future applications. Although the GMDS system was never fully developed, it did provide proof of concept and gave RTIO a clear vision of what should be included in an aggregator tool to provide a one-stop geotech shop for practitioners.

\subsection{Vendor evaluation}

With sustainability of an in-house developed tool presenting many business challenges, the decision was made to explore the available external options. Four different vendors were invited to submit proposals for a RTIO solution and provide a demonstration of application functionality. Three proposals were ultimately received and reviewed by a team comprising site geotechnical personnel and managers, and Perth geotechnical engineers. A set of technical and commercial evaluation criteria were developed including capabilities, graphing and reporting, ease of use, company characteristics and pricing (Table 1). Weightings were applied to both main categories and subcategories. Capability was deemed to be the most important, with ease of use and costs next. Graphing/reporting and company evaluation was the lowest importance. At this time, a decision was already made not to pursue the in-house developed product and it was hence not included in the evaluation. The available off-the-shelf products were clearly in a more advanced state than the in-house product. 
Table $1 \quad$ Vendor evaluation criteria

\begin{tabular}{|c|c|}
\hline Evaluation criteria & Metric \\
\hline \multirow[t]{9}{*}{ Capabilities } & Authority levels \\
\hline & Validation auditing \\
\hline & Concurrent users (including internet) \\
\hline & Data from all monitoring devices accepted \\
\hline & Accepts data from various software/databases \\
\hline & Inclusion and representation of non-temporal data \\
\hline & Management of photos \\
\hline & Dashboard showing health and alarming \\
\hline & 2D or 3D GIS visualisation \\
\hline \multirow[t]{5}{*}{ Graphing and reporting } & Graphing multiple instruments and prisms \\
\hline & Customisation/diversity of graphs \\
\hline & Report auto-generation \\
\hline & Visual selection of devices to graph \\
\hline & Velocity information from displacement graph \\
\hline \multirow[t]{5}{*}{ Ease of use } & Visually appealing \\
\hline & Customisable screen \\
\hline & User-friendly software \\
\hline & Sub clicks \\
\hline & Speed of software \\
\hline \multirow[t]{6}{*}{ Company characteristics } & Ability to implement on time \\
\hline & Ability to support (location) \\
\hline & Synergies with other Rio Tinto operations \\
\hline & Willingness to customise software \\
\hline & Training \\
\hline & Company size \\
\hline \multirow[t]{4}{*}{ Pricing } & Pilot programme \\
\hline & Cost of package \\
\hline & Support plan \\
\hline & Licencing fees \\
\hline
\end{tabular}

Each of the applications presented had strengths and weaknesses against the criteria, with vendor 2 emerging as the preferred option (Figure 3 ). Vendor 1 demonstrated a relatively new software that was easy to use with good graphics and demonstrated potential. However, the application currently did not have the RTIO desired functionality, as the vendor focus was on analysis of mostly non-temporal data. This application did not have the ability to display and interpret radar data. Vendor 3 focussed mostly on data collection, and less on interpretation. This application could not plot any radar data and only had accesses to IBIS radar data for display as a .dxf file.

Vendor 2 presented a more mature application that was currently used by three other Rio Tinto operations globally. The application met many of the RTIO functionality requirements including the ability to display data from all three radar vendors. This was selected as the best-fit application to then work with RTIO to address remaining gaps including inclusion of georeferenced events and documents (i.e. hazard map, GRAHAMS risk 
map, fall of ground, photos), inclusion of non-georeferenced information, improved graphing functionality and development of a customisable dashboard for system health. With three other Rio Tinto global operations also using the application, there was an opportunity for collaboration to ensure common requirements were fulfilled.

\section{Comparison of vendors per evaluation criteria}

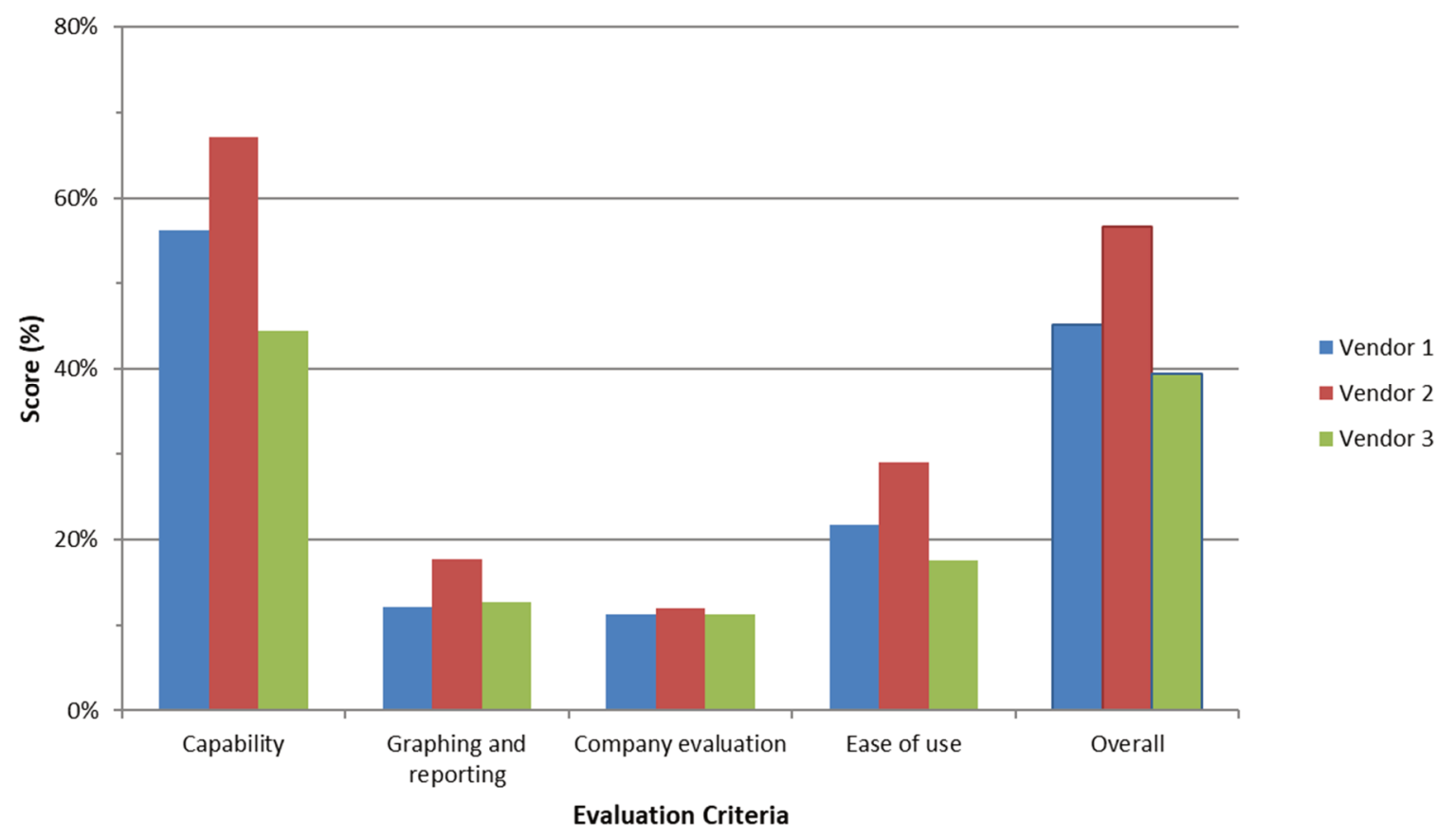

Figure 3 Summary comparison of vendors per evaluation criteria

\section{$4 \quad$ Addressing the gaps}

During the development of the GMDS system, the RTIO team developed a strong vision of what a comprehensive geotechnical aggregator system should consist of. The focus was not only on temporal (i.e. monitoring) data but also included non-temporal and non-georeferenced data. The system should provide access to all geotechnical data and information required to manage geotechnical risks.

The systems that were reviewed during the evaluation process mostly focussed on temporal data. The software of the preferred vendor had most of the temporal data features required by RTIO available in their product. A number of key additional features were identified and discussed with the vendor. Examples and prototypes of expectations were provided to address the identified gaps.

\subsection{Georeferenced temporal data}

Improved graphing: One of the main purposes of a slope monitoring system is to monitor how large sections of a slope performs in response to mining. This is often done by monitoring a group of prisms, as opposed to focussing on a single prism. Although the software had the ability to graph a group of prisms, it was a slow process. Once the group of prisms were set up, the graph and settings (e.g. details of prisms, time periods, etc.) could not be saved. It was also not possible to transfer the graph to the 'Report' section of the software. Analyses started by a user could potentially be lost. RTIO worked with the vendor to develop the 'Group Sensor' function, which enables a user to set up a group of sensors, which can be easily accessed and interrogated on future occasions. Graphs that were created in graph view mode can now be dragged and dropped into the report section, allowing graphs to be saved for future reference.

Displacement period velocity: RTIO identified a discrepancy between the method used by some prism vendor software, and the traditional method used by geotechnical engineers to determine velocity (refer to 
Figure 4). In the Trimble T4D software used by RTIO, the 3D or 2D velocity is calculated by first determining the velocity in directional components (i.e. northing and easting). The resultant 3D or 2D velocity is then calculated by using the velocities for each component in the Pythagoras theorem formula. Traditionally, geotechnical engineers used displacement graphs to calculate velocity, where the amount of displacement is read off the graph and divided by the time period over which the displacement occurred (the trend line method). In a specific slope monitoring example in RTIO, the software reported a velocity of $5.7 \mathrm{~mm} /$ day, where the trend line method resulted in a velocity of $1 \mathrm{~mm} /$ day. Such a discrepancy can lead to confusion, especially if monitoring alarms are based on velocity. The aggregator software used the same method as Trimble T4D to determine velocity. At the request of RTIO, the vendor introduced 'Displacement Period Velocity', which is the trend line method to determine velocity.

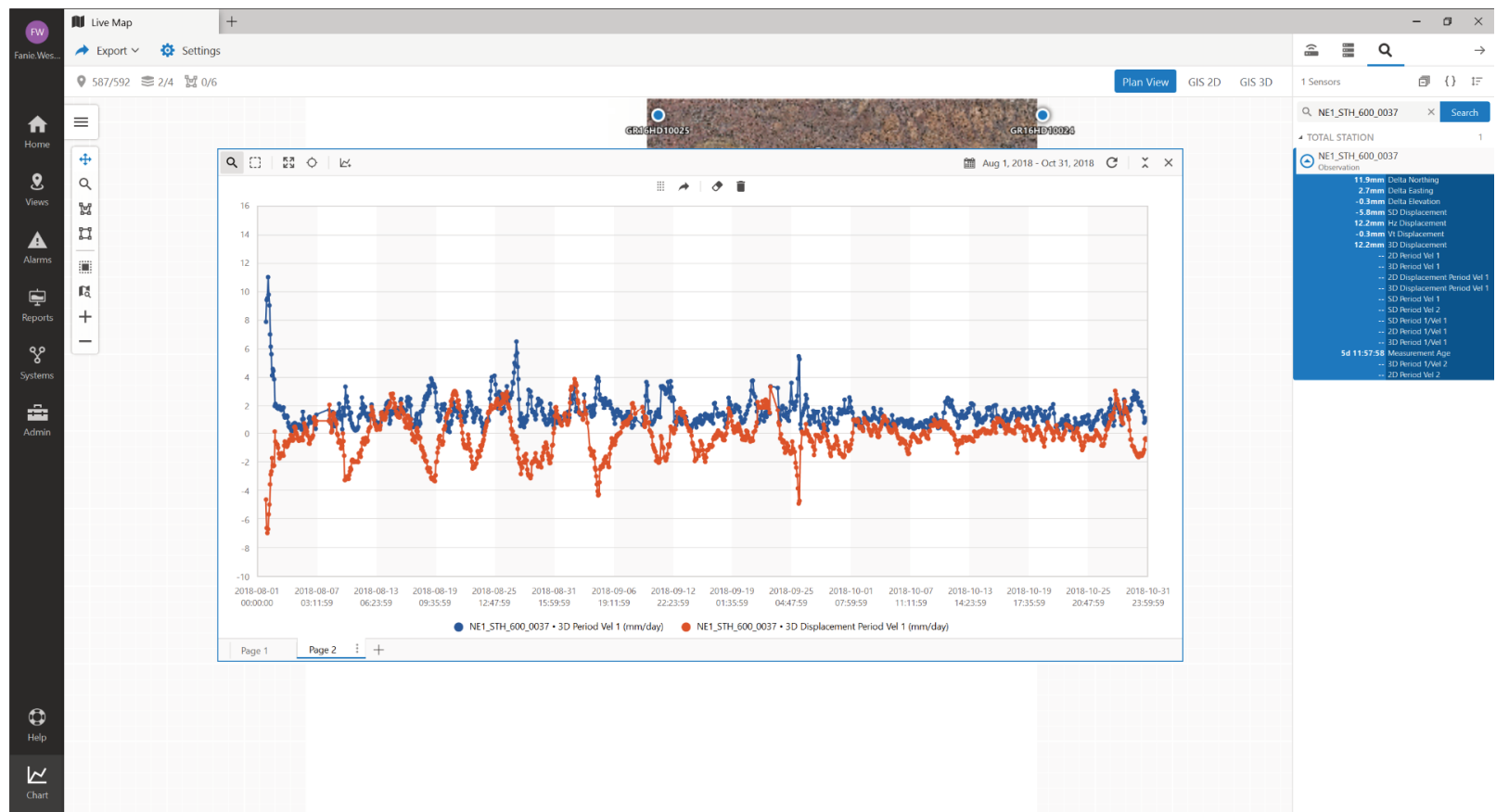

Figure 4 Graph from aggregator software which shows the difference between period velocity and displacement velocity

Alignment with prism software data: Whilst trialling the aggregator software, RTIO identified a discrepancy between the monitoring data as reported by Trimble T4D and the aggregator software. At RTIO, the Trimble T4D system was already well-entrenched in the workflow of the geotechnical engineer, with a customised alarming system developed to enable alarming through the truck dispatch system to the operations centre in Perth. For these reasons, it is envisaged that both systems will be utilised by RTIO in the future. For obvious reasons, it is important that the two systems deliver the same data for analysis. RTIO worked closely with the vendor to identify the sources of these discrepancies and address them to ensure that both software systems deliver the same results.

\subsection{Georeferenced, non-temporal data}

The intent of the aggregator system is to host more than temporal data. A user should also be able to access non-temporal data, both georeferenced and non-georeferenced. Examples of non-temporal, georeferenced data that needs to be accessed are photographs, geotechnical risk and geotechnical hazard information.

Photographs: Geotechnical engineers often make use of photographs to monitor the condition of slopes. Photos are taken from the same vantage point on either a regular or an ad hoc interval. The photos can then be used to compare the condition of the slope and identify deterioration with time, or just serve as a visual record on the progress of the slope with time. Storage and management of these photos can be tedious and time-consuming work. Photos can be either stored by location or chronologically. This has the advantage of 
being easy to scroll through and view the photos, but takes some time to store the photos in the correct directory/locations. The other option can be to store the photos chronologically, which makes storing easy but viewing more time-consuming, especially if the photos were not renamed according to location.

RTIO requested the vendor to develop a function whereby the photo locations are shown in the 'Live View' option of the software (Figure 5). Small photo thumbnails visible in the map view are displayed at the bottom of the map. Any number of individual photos can be selected and viewed, which will show the changes in a specific area as time progresses. If photos are taken with a GPS-enabled camera, all the photos can be stored in a single location, eliminating the requirement to sort and store photos. Non-georeferenced photos have to be manually placed in the correct location, but this is a once-off exercise and can be accessed in a similar manner as georeferenced photos after this. This function makes the management of photographs much easier, less time-consuming, and much more accessible.

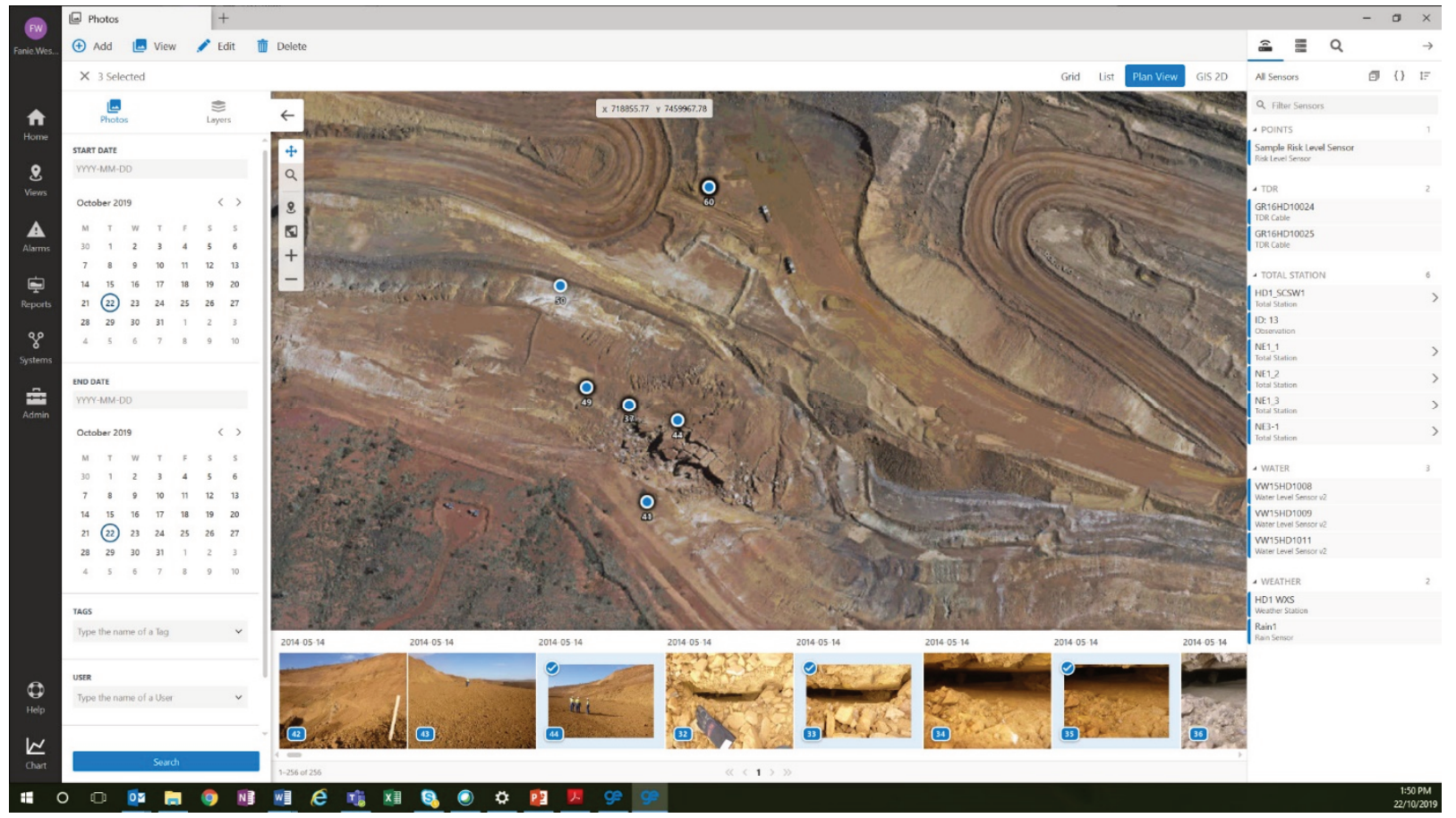

Figure 5 The photo manager feature in the aggregator software. The location of the photos are shown in the main view and thumbnail images of the photos displayed at the bottom. Individual photos can be selected and viewed in full screen view

Slope performance documents: The use and importance of non-temporal geotechnical information, summarised in documents, was discussed earlier. Since the RTIO vision is to have all geotechnical data available in one location, these documents needed to be linked to a point, line or polygon in the software. A linked document is accessible by selecting the relevant icon. The colour of an icon can also provide further information about the document, e.g. the risk level associated with a hazard in the pit can be indicated by the colour of the icon. The ability to visualise the location and detail of slope performance documents side by side with temporal monitoring data in one platform can assist to understand and monitor slope performance. Although none of these hazard management and slope performance functions have been fully developed at the time of writing, proto formats and mock-ups of required information were developed and development is in progress.

\subsection{Non-georeferenced information}

RTIO has 16 mine operations across the Pilbara. A mine operation is often subdivided into different mining areas with a number of open pits in each mining area. In order to have visibility of the geotechnical risks and monitoring status, the need was identified to report summary geotechnical parameters on a dashboard as a Pilbara wide summary, but also on a mine operation, mining area and pit level. This feature will give a user 
the option to either look at high level summary data (e.g. average slope radar availability across the Pilbara) or drill down to the details related to a specific monitoring device in a specific pit (e.g. availability of a specific slope radar in a specific pit). Both temporal (e.g. monitoring data) and non-temporal data (e.g. risk level) will be reported in the dashboard.

In addition to providing summary data of geotechnical parameters, it is envisaged that the Geotechnical Dashboard will also provide quick links to key systems and documents needed by the geotechnical engineer to manage geotechnical risks onsite. A simplified version of this concept was implemented in the RTIO GMDS system. Although the vendor has demonstrated this ability to develop this feature with a prototype 'Smart Search' it still has to be fully developed and implemented.

\section{Conclusion}

The geotechnical engineer has a wide range of information and data available to assist with the management of geotechnical risks at a mine site, and the list continues to grow. This data is often stored in a number of locations and accessed through different platforms and software. Finding all of the appropriate information can be challenging and time-consuming. The development of a single platform where the bulk of this information can be accessed, will not only make more efficient use of the geotechnical engineers time but will also provide a more holistic view and understanding, improving decision-making and enhancing geotechnical risk management.

During the development of an in-house data aggregator platform, RTIO has built up a clear vision of what a complete 'one-stop geotech shop' platform should consist of. It also highlighted the challenges related to the development and sustaining of a fully functioning platform. RTIO, in cooperation with the vendor of an off-the-shelf product, made progress towards addressing the remaining system requirements. The most urgent temporal data requirements have now been addressed and the photography functionality has been a big enhancement. The focus will now be on the document links to geometries function, with the dashboard to follow.

\section{Acknowledgement}

The authors would like to acknowledge their RTIO colleagues who contributed and assisted with this project to date, as well as the Navstar personnel for their support and cooperation. The permission from Rio Tinto to publish this work is gratefully appreciated.

\section{References}

Bar, N, Reynolds, M, Nicoll, S \& Bran, D 2018, 'Geotechnical data management and visualization systems: meeting the data challenge of the 21st century and maximizing value for open pit mines', in V Litvinenko (ed.), Proceedings of Eurock 2018: Geomechanics and Geodynamics of Rock Masses, St Petersburg Mining University, St Petersburg, pp. 973-978.

Conrad, WJ \& Neuwirt, AM 2019, 'Using an integrated monitoring platform to communicate geotechnical risk to project stakeholders', in J Wesseloo (ed.), Proceedings of the First International Conference on Mining Geomechanical Risk, Australian Centre for Geomechanics, Perth, pp. 171-180.

de Graaf, PJH \& Wessels, SDN 2013, 'Slope monitoring and data visualisation state-of-the-art - advancing to Rio Tinto Iron Ore's Mine of the Future ${ }^{T M}$ ', in PM Dight (ed.), Proceedings of the 2013 International Symposium on Slope Stability in Open Pit Mining and Civil Engineering, Australian Centre for Geomechanics, Perth, pp. 803-814.

de Graaf, PJH \& Wessels, SDN 2016, 'A framework for managing geotechnical risk across multiple operations', The Journal of The Southern African Institute for Mine and Metallurgy, vol. 116, no. 5, pp. 367-377.

Girard, JM 2001, 'Assessing and monitoring open pit mine highwalls', Proceedings of the 32nd Annual Institute of Mining Health, Safety and Research, National Institute for Occupational Safety \& Health, Spokane.

Girard, JM \& McHugh, E 2000, 'Detecting problems with mine slope stability', Proceedings of the 31st Annual Institute on Mining Health, Safety, and Research, National Institute for Occupational Safety \& Health, Spokane.

Sjöberg, J 1996, Large scale slope stability in open pit mining, Technical Report 1996, Luleå University of Technology, Luleå.

Wessels, SDN \& Dixon, R 2019, 'Managing geotechnical risk in multi-pit operation', in J Wesseloo (ed.), Proceedings of the First International Conference on Mining Geomechanical Risk, Australian Centre for Geomechanics, Perth, pp. 311-322. 